\title{
Classifier-Independent Feature Selection Based on Non-parametric Discriminant Analysis
}

\author{
Naoto Abe ${ }^{1}$, Mineichi Kudo ${ }^{1}$, and Masaru Shimbo ${ }^{2}$ \\ 1 Division of Systems and Information Engineering \\ Graduate School of Engineering \\ Hokkaido University, Sapporo 060-8628, Japan \\ \{chokujin,mine\}@main.eng.hokudai.ac.jp \\ ${ }^{2}$ Faculty of Information Media, Hokkaido Information University \\ Ebetsu 069-8585, Japan \\ shimbo@do-johodai.ac.jp
}

\begin{abstract}
A novel algorithm for classifier-independent feature selection is proposed. There are two possible ways to select features that are effective for any kind of classifier. One way is to correctly estimate the class-conditional probability densities and the other way is to accurately estimate the discrimination boundary. The purpose of this study is to find the discrimination boundary and to determine the effectiveness of features in terms of normal vectors along the boundary. The fundamental effectiveness of this approach was confirmed by the results of several experiments.
\end{abstract}

\section{Introduction}

Feature selection is a procedure to find a feature subset that has the most discriminative information from an original feature set. In large-scale problems with over 50 features, there may exist garbage features that have an adverse effect on construction of classifiers. In such a case, it is expected that the performance of classifiers can be improved by removing such garbage features.

Many algorithms for feature selection have been proposed. Many references to report presenting such algorithms are given in [1]. These algorithms can be divided into two groups. One group is called classifier-specific feature selection algorithms [1], where the goodness of a feature subset is measured in terms of the estimated performance of a certain classifier. These algorithms are useful when it is known in advance what classifier is used. However, it is more desirable to select a feature subset that is effective for any kind of classifier. Therefore, another group of algorithms, called classifier-independent feature selection algorithms $[2,3,4]$, has been studied, and their criterion functions are connected with estimates of the recognition rate of the Bayes classifier. Algorithms belonging to the latter group can further be divided into two groups: one group of algorithms designed to estimate class-conditional densities or a distributional structure $[2,3]$ and another group of algorithms designed to estimate discrimination boundaries $[4,5]$. When we have only a small training sample set, estimating 


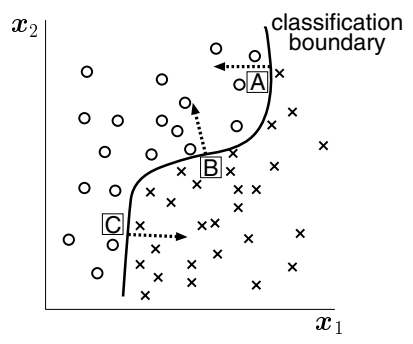

Fig. 1. Local importance of features

the discrimination boundary seems to be better than estimating densities. In this paper, we examine the effectiveness of such a trial.

\section{Discriminative Information Based on Non-parametric Discriminant Analysis}

\subsection{Feature Importance Based on Classification Boundary}

Our approach is twofold: (1) to estimate the discrimination boundary as precisely as possible and (2) to evaluate the effectiveness of features in terms of the boundary. First, we notice that the normal vectors along the discrimination boundary show which features are necessary for discriminating two classes (Fig. 1). A vector at point $\mathrm{A}$ or $\mathrm{C}$ indicates that feature $x_{1}$ is important, and feature $x_{2}$ is important at point $\mathrm{B}$. Thus, it can be seen that these normal vectors reflect the importance of features locally. Since what we want is a feature subset that is effective globally, we find all necessary features by combining such local evidence.

\subsection{Non-parametric Discriminant Analysis}

Here, let us consider two-class $\left(\omega_{1}\right.$ and $\left.\omega_{2}\right)$ problems first. The method described below can easily be extended to multi-class problems. The normal vectors along the discrimination boundary can be estimated using non-parametric discriminant analysis proposed by Fukunaga and Mantock [6]. In this analysis, $k$ nearest unlike neighbors (NUNs) taken from the opposite class play a main role. For a sample $\boldsymbol{x} \in \omega, k$ nearest unlike neighbors $\boldsymbol{y}_{1}, \ldots, \boldsymbol{y}_{k} \in \omega^{\prime} \neq \omega$, where $\left\|\boldsymbol{y}_{1}-\boldsymbol{x}\right\| \leq$ $\left\|\boldsymbol{y}_{2}-\boldsymbol{x}\right\| \leq \cdots \leq\left\|\boldsymbol{y}_{k}-\boldsymbol{x}\right\|$, are found. Then, a normal vector $\boldsymbol{v}_{\boldsymbol{x}}$ at $\boldsymbol{x}$ is formulated as

$$
\boldsymbol{v}_{\boldsymbol{x}}=\frac{1}{k}\left(\sum_{i=1}^{k} \boldsymbol{y}_{i}\right)-\boldsymbol{x}
$$


Also, vector $\boldsymbol{v}_{\boldsymbol{x}}$ is weighted by

$$
w_{v}=\frac{\min \left\{\left\|\boldsymbol{y}_{k}-\boldsymbol{x}\right\|,\left\|\boldsymbol{x}_{k}-\boldsymbol{x}\right\|\right\}}{\left\|\boldsymbol{y}_{k}-\boldsymbol{x}\right\|+\left\|\boldsymbol{x}_{k}-\boldsymbol{x}\right\|} .
$$

Here, $\boldsymbol{x}_{k}$ is the $k$ th NN of $\boldsymbol{x}$ taken from the same class $\omega$. This weight takes a value close to 0.5 when $\boldsymbol{x}$ is close to the discrimination boundary and declines to zero as $\boldsymbol{x}$ moves away from the discrimination boundary. Fukunaga and Mantock used these vectors with weights to calculate a non-parametric form of a betweenclass covariance matrix.

\section{Proposed Method}

\subsection{Modified Normal Vectors}

There is a problem in calculation (1) of weights for the normal vectors. Such an example is shown in Fig. 2. As shown in Fig. 2(a), if we use weighting formula (1), when the distances $\left\|\boldsymbol{y}_{k}-\boldsymbol{x}\right\|$ and $\left\|\boldsymbol{x}_{k}-\boldsymbol{x}\right\|$ are almost the same, such a point $\boldsymbol{x}$ is treated as being located near the discrimination boundary, which results in $w_{v} \doteq \frac{1}{2}$. As a result, a normal vector for such a point can show a wrong direction with a high weight close to the maximum weight $\frac{1}{2}$. To cope with this problem, we calculate the normal vectors as

$$
\boldsymbol{v}_{\boldsymbol{x}}=\sum_{i=1}^{k} e^{-\sigma\left\|\boldsymbol{y}_{i}-\boldsymbol{x}\right\|} \frac{\boldsymbol{y}_{i}-\boldsymbol{x}}{\left\|\boldsymbol{y}_{i}-\boldsymbol{x}\right\|}
$$

Here, $\sigma(\sigma>0)$ is a control parameter. In this way, as shown in Fig. 2(b), vectors $\left(\boldsymbol{y}_{i}-\boldsymbol{x}\right)$ 's cancel their bad influence by themselves. Two examples of normal vectors calculated in this way are shown in Fig. 3.

\subsection{Evaluation of Features}

To combine the local importance of features, we take a simple way. That is, for calculated normal vectors $\boldsymbol{v}_{\boldsymbol{x}}=\left(v_{1 x}, v_{2 x}, \ldots, v_{D x}\right)$ of class $\omega_{c}$, we sum up the absolute values of $i$ th component $v_{i x}$ to have the importance measure $f_{i}^{c}=$ $1 / n_{c} \sum_{\boldsymbol{x} \in \omega_{c}}\left|v_{i x}\right|$, and take the average over classes as $f_{i}=\sum_{c=1}^{C} P_{c} f_{i}^{c}$. Here, $D$ is the dimensionality, $n_{c}$ is the number of samples from $\omega_{c}$, and $P_{c}$ is a priori probability of class $\omega_{c}$ which is estimated by $P_{c}=n_{c} / \sum_{c=1}^{C} n_{c}$. Last, we normalize $f_{i}$ as $f_{i} \leftarrow f_{i} / \sum_{i=1}^{D} f_{i}$.

\subsection{Determination of Size}

In classifier-independent feature selection, it is important to remove only garbage features. If the number of training samples is sufficiently large, the feature importance measure of a garbage feature is expected to take a very small value. 


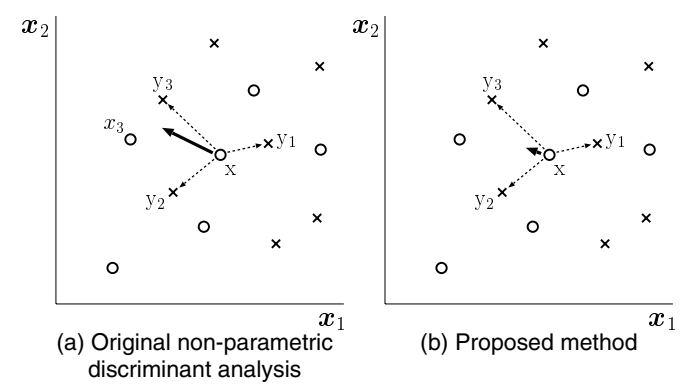

Fig. 2. Calculation of weights for normal vectors when $k=3$
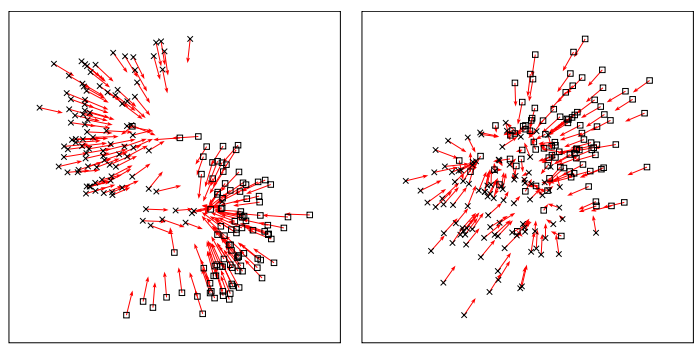

Fig. 3. Two examples of normal vectors calculated by the proposed method $(\mathrm{k}=4)$

Therefore, we use a function $J(d)=1-\min _{1 \leq i \leq d} f_{i}$ for evaluating the goodness of a feature subset of size $d(d=1,2, \ldots, D)$. In an ideal case in which $d^{*}\left(d^{*}<D\right)$ features are garbage and the remaining $D-d^{*}$ features contribute equally to the performance, we have a criterion curve as shown in Fig. 4(a). In this case, we can remove all garbage features with $\theta<1 / d^{*}$. However, in a practical case in which the number of training samples is limited, even garbage features may show a little contribution. In this case, we use a threshold $\theta$ in order to determine the size $d_{\theta}$. That is, we find the $\theta$-degradation point of the maximum value $J(D)$ (Fig. 4(b)). The proposed algorithm is summarized as follows:

Step 0: Set the value of threshold $\theta>0$. Let $\mathrm{d}=\mathrm{D}$.

Step 1: Calculate the normal vectors $\boldsymbol{v}_{\boldsymbol{x}}$ with respect to every sample $\boldsymbol{x}$ by Eq. (2).

Step 2: Calculate feature importance $f_{i}(i=1,2, \ldots, d)$. If $d=D$, set $J(D)$ for $J_{\max }$.

Step 3: Remove the worst feature $f_{i}=\arg \min _{i} f_{i}$.

Step 4: If $J_{d}<(1-\theta) J_{\max }$, terminate the algorithm and output the subset. Otherwise, with $d=d-1$, go to step 1 . 


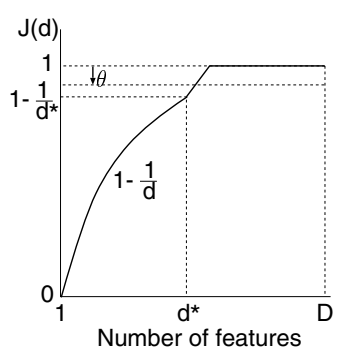

(a) Ideal case

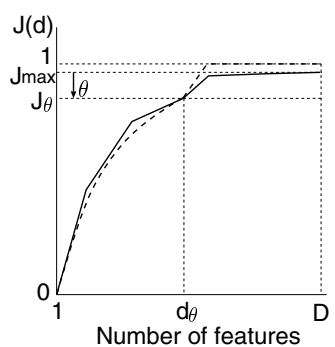

(b) Practical case

Fig. 4. Determination of size

\section{Experiments}

Two artificial datasets and one real dataset were used in the experiments [9]. In the experiments, $\theta$ was taken as $1 \%$ or $5 \%$. Here, $\theta=1 \%$ was chosen from the viewpoint of removing only garbage features. The value of $5 \%$ was taken from the practical viewpoint of choosing a smaller feature subset at the expense of a slight degradation of discriminative information. A simple experiment was carried out to determine the appropriate values of $k$ and $\sigma$. A value two-times greater than the number of features was chosen for $k$ and 0.1 was chosen for $\sigma$. Six classifiers were used to evaluate the goodness of a selected feature subset: the Bayes linear classifier (LNR), the Bayes quadratic classifier (QDR), the C4.5 decision tree classifier [7] (C45), the one-nearest neighbor (1NN) classifier, the subclass classifier [8] (SUB), and the neural network classifier (NRL). The recognition rates were calculated by the 10 -fold cross validation technique.

\section{Friedman: A Friedman database [4].}

In this database, there are two classes, $\omega_{1}$ and $\omega_{2}$. The samples of $\omega_{1}$ were generated according to a Gaussian with a unit covariance matrix and zero mean. The samples of $\omega_{2}$ surround those of $\omega_{1}$ in the first four features that are distributed uniformly within a four-dimensional spherical slab centered at the origin with an inner radius 3.5 and outer radius 4.0 in the samples of $\omega_{2}$. The last six features of $\omega_{2}$ are distributed as a Gaussian with a unit covariance matrix and zero mean. Each class has 100 samples.

2. Waveform: A waveform database [9].

In this database, there are three classes and 40 features with continuous values between 0 and 6 . Each class is a random combination of two triangular waveforms with noise added. Each class is generated from a combination using two of three base waves. The instances are generated such that features $1,2, \ldots, 21$ are necessary for class separation, whereas 
features $22,23, \ldots, 40$ have random variables. The numbers of samples were 67 for class 1, 54 for class 2, and 79 for class 3 , respectively.

\section{Sonar: A sonar database [9].}

The task was to discriminate between sonar signals bounced off a metal cylinder and those bounced off a roughly cylindrical rock using 60 features, each of which describes the energy within a particular frequency band, integrated over a certain period of time. The database consists of 111 patterns obtained by bouncing sonar signals off a metal cylinder at various angles and under various conditions and 97 patterns obtained from rocks under similar conditions.

The results were evaluated mainly from the following two viewpoints.

- Compared with the case in which all original features are used, if all garbage features are successfully removed, the recognition rates of all classifiers would be improved or maintained. We examined whether this is the case or not.

- Since the classifier that shows the best performance among all classifiers can be considered as being closest to the Bayes classifier, we examined whether the number of selected features is larger than that corresponding to the peak of the best classifier or not.

Unlike classifier-specific feature selection, what we hope for is a minimal feature subset that includes all discriminative features in classifier-independent feature selection. Thus, a slightly larger feature subset that includes them is acceptable. For comparison with the density estimation approaches, we carried out the same experiments using the divergence method [10], which is based on an axis-parallel Gaussian mixture model. In the divergence method, a feature subset of a given size that maximizes Kullback-Leibler divergence is selected on the basis of estimated densities. Changing the size from $D$ down to 1 , we found a sequence of feature subsets and selected a feature subset using a threshold ( $\alpha=1 \%$ or $5 \%$ ) as the proposed method does. For details, see [10].

The results of the experiments are shown in Figs. 5-7. Compared with the case that all the features were used, the recognition rates of the six classifiers were all either improved or maintained by using the proposed method, as well as the divergence method. Therefore, the proposed method is effective in the first viewpoint of evaluation. In addition, the results with $\theta=1 \%$ were satisfactory even from the second viewpoint of evaluation.

Next, we examined the difference between the recognition rates using the proposed method and the divergence method. The difference between the recognition rates is shown in Fig. 8. In this figure, only the best three classifiers are shown. For the Friedman dataset, there was no notable difference between recognition rates. However, for the Waveform and Sonar datasets, a slight improvement in the recognition rate was obtained by using the proposed method. 


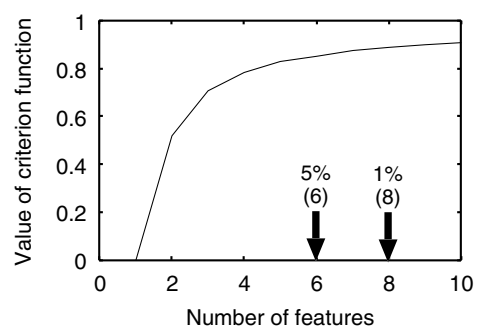

(a) Criterion curve of the proposed method

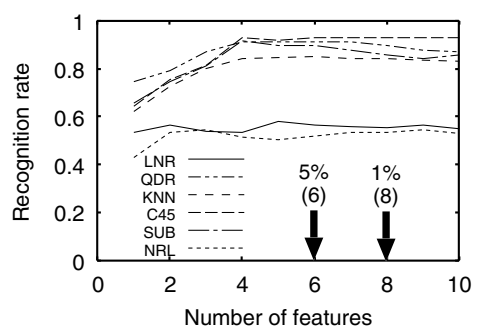

(c) Recognition rate using feature subsets by the proposed method

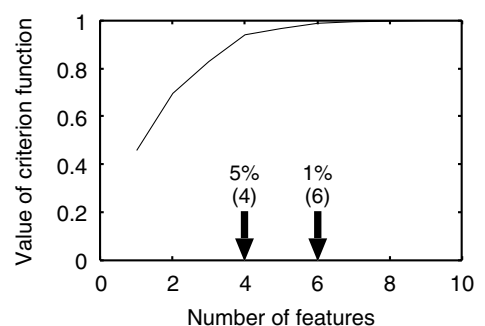

(b) Criterion curve of the divergence method

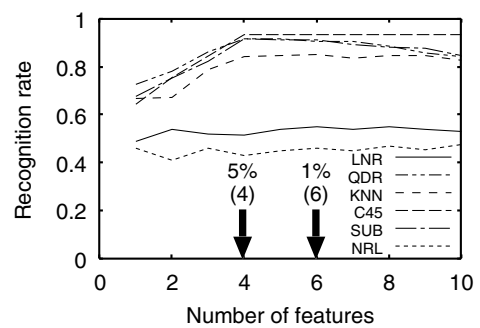

(d) Recognition rate using feature subsets by the divergence method

Fig. 5. Results of Friedman data. The values in parentheses are the numbers of selected features

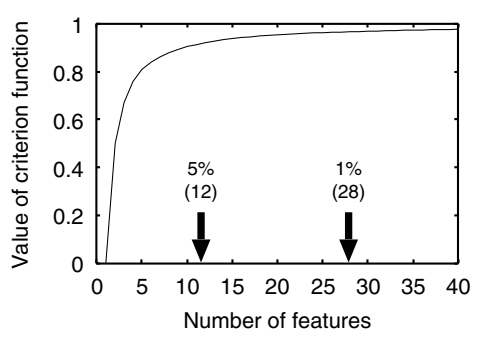

(a) Criterion curve of the proposed method

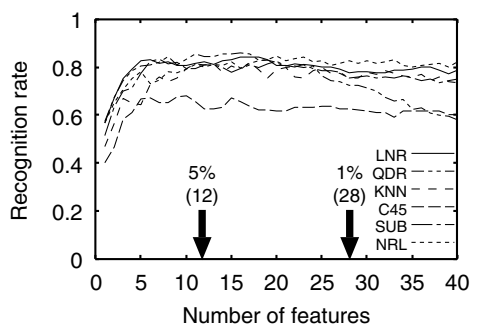

(c) Recognition rate using feature subsets by the proposed method

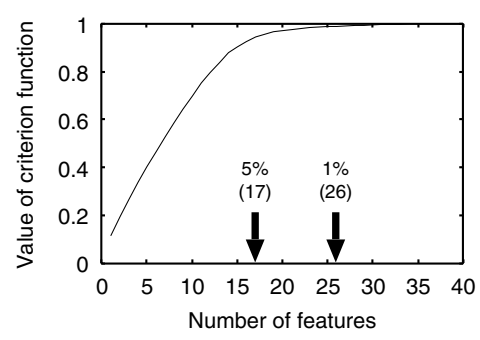

(b) Criterion curve of the divergence method

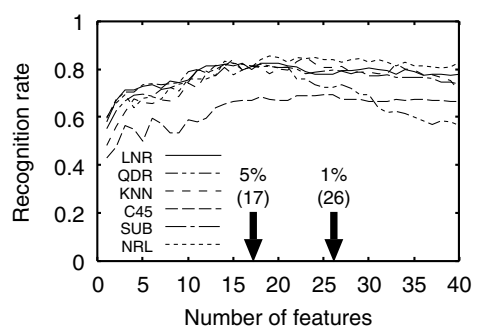

(d) Recognition rate using feature subsets by the divergence method

Fig. 6. Results of Waveform data. The values in parentheses are the numbers of selected features 


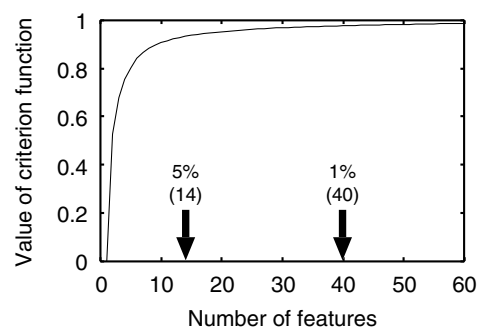

(a) Criterion curve of the proposed method

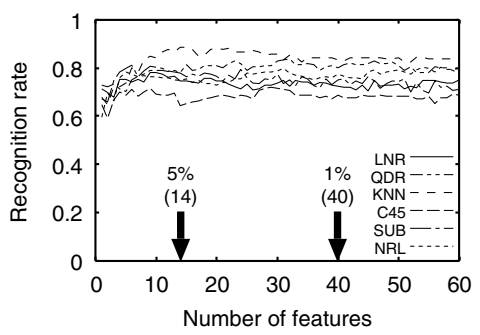

(c) Recognition rate using feature subsets by the proposed method

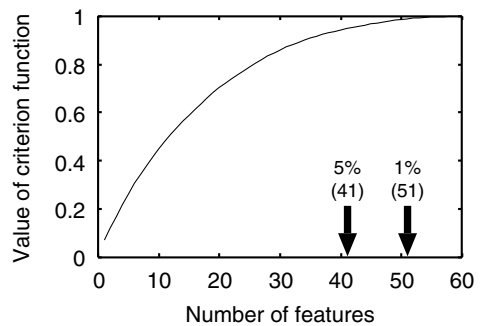

(b) Criterion curve of the divergence method

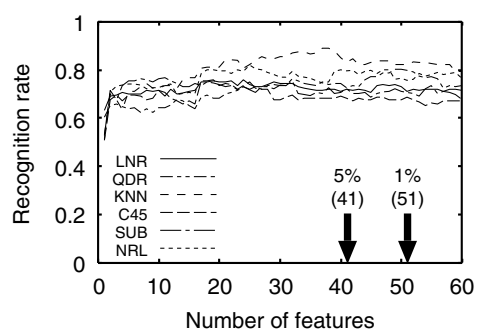

(d) Recognition rate using feature subsets by the divergence method

Fig. 7. Results of Sonar data. The values in parentheses are the numbers of selected features

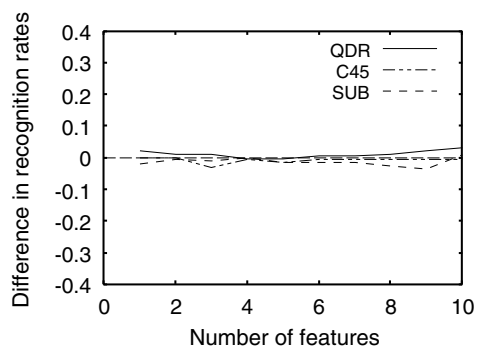

(a) Friedman

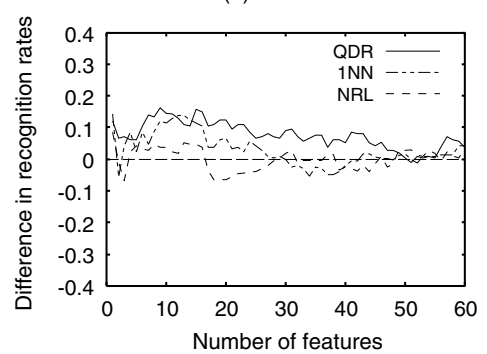

(c) Sonar

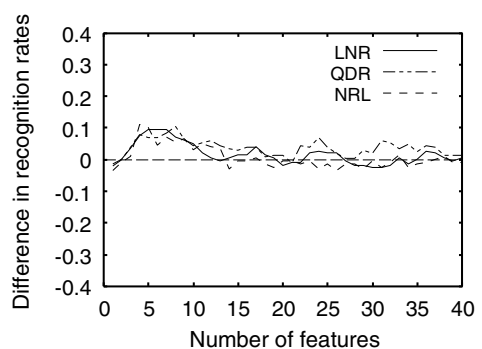

(b) Waveform

Fig. 8. Difference in recognition rates (A)-(B), where (A) is the recognition rate of the proposed method and $(\mathrm{B})$ is that of the divergence method. Only the best three classifiers are shown 


\section{Discussion}

The curve of the criterion function $J(d)(d=1,2, \ldots, D)$ obtained by the proposed method is almost proportional to that of the recognition rates of many classifiers. This means that the proposed method is better than the divergence method for evaluation of feature subsets. For example, see Fig. 6. In the divergence method, many parameters must be estimated appropriately in the probability density. On the other hand, the proposed method requires only two parameters, a control parameter of the normal vectors and a threshold for determining the size of a feature subset to be selected. In addition, the discrimination boundary is expected to be learned faster than the densities of classes. Unfortunately, we have not carried out any experiments to confirm this point, it is worth examining if this is true or not by dealing different sizes of training samples.

\section{Conclusion}

We have proposed an algorithm for classifier-independent feature selection using non-parametric discriminant analysis. The fundamental effectiveness of the proposed method was confirmed by results of experiments using two artificial datasets and one real dataset. Overall, the effectiveness of the proposed method is comparable with that of the divergence method and, the proposed method is superior in simplicity of the parameter setting.

\section{References}

1. Kudo, M., and Sklansky J.: Comparison of Algorithms that Select Features for Pattern Classifiers. Pattern Recognition 33-1 (2000) 25-41 470

2. Holz, H. J., and Loew, M. H.: Relative Feature Importance: A ClassifierIndependent Approach to Feature Selection. In: Gelsema E. S. and Kanal L. N. (eds.) Pattern Recognition in Practice IV, Amsterdam: Elsevier (1994) 473-487 470

3. Novovičová, J., Pudil, P., and Kittler, J.: Divergence Based Feature Selection for Multimodal Class Densities. IEEE Transactions on Pattern Analysis and Machine Intelligence 18 (1996) 218-223 470

4. Kudo, M., and Shimbo, M.: Feature Selection Based on the Structural Indices of Categories. Pattern Recognition 26 (1993) 891-901 470, 474

5. Egmont-Petersen, M., Dassen, W. R. M., and Reiber, J. H. C.: Sequential Selection of Discrete Features for Neural Networks - A Bayesian Approach to Building a Cascade. Pattern Recognition Letters 20 (1999) 1439-1448 470

6. Fukunaga, K., and Mantock, J. M.: Nonparametric Discriminant Analysis. IEEE Transactions on Pattern Analysis and Machine Intelligence 5 (1983) 671-678 471

7. Quinlan, J. R.: C4.5: Programs for Machine Learning. Morgan Kaufmann San Mateo CA (1993) 474

8. Kudo, M., Yanagi, S., and Shimbo, M.: Construction of Class Region by a Randomized Algorithm: A Randomized Subclass Method. Pattern Recognition 29 (1996) 581-588 474 
9. Murphy, P. M., and Aha, D. W.: UCI Repository of machine learning databases [Machine-readable data repository]. University of California Irvine, Department of Information and Computation Science (1996) 474, 475

10. Abe, N., Kudo, M., Toyama, J., and Shimbo, M.: A Divergence Criterion for Classifier-Independent Feature Selection. In: Ferri, F. J., Inesta, J. M., Amin, A., and Pudil, P. (eds.) Advances in Pattern Recognition, Lecture Notes in Computer Science, Alicante, Spain, (2000) 668-676 475 